\title{
Analysis of a hybrid TATA box binding protein originating from mesophilic and thermophilic donor organisms
}

\author{
Annette Kopitz $^{\mathrm{a}, \mathrm{b}}$, Jörg Soppa ${ }^{\mathrm{b}}$ and Karin Hauser ${ }^{\mathrm{a}, \mathrm{c}, *}$ \\ ${ }^{a}$ Institute of Biophysics, Goethe-University Frankfurt, Frankfurt am Main, Germany \\ ${ }^{\mathrm{b}}$ Institute of Molecular Biosciences, Goethe-University Frankfurt, Frankfurt am Main, Germany \\ ${ }^{\mathrm{c}}$ Institute of Physics, RWTH Aachen University, Aachen, Germany
}

\begin{abstract}
The TATA Box Binding Protein (TBP) is a $20 \mathrm{kD}$ protein that is essential and universally conserved in eucarya and archaea. Especially among archaea, organisms can be found that live below $0^{\circ} \mathrm{C}$ as well as organisms that grow above $100^{\circ} \mathrm{C}$. The archaeal TBPs show a high sequence identity and a similar structure consisting of $\alpha$-helices and $\beta$-sheets that are arranged in a saddle-shape 2-symmetric fold. In previous studies, we have characterized the thermal stability of thermophilic and mesophilic archaeal TBPs by infrared spectroscopy and showed the correlation between the transition temperature $\left(T_{\mathrm{m}}\right)$ and the optimal growth temperature (OGT) of the respective donor organism. In this study, a "new" mutant TBP has been constructed, produced, purified and analyzed for a deeper understanding of the molecular mechanisms of thermoadaptation. The $\beta$-sheet part of the mutant consists of the TBP from Methanothermobacter thermoautotrophicus (OGT $65^{\circ} \mathrm{C}$, MtTBP65) whose $\alpha$-helices have been exchanged by those of Methanosarcina mazei (OGT $\left.37^{\circ} \mathrm{C}, \mathrm{MmTBP} 37\right)$. The Hybrid-TBP irreversibly aggregates after thermal unfolding just like MmTBP37 and MtTBP65, but the $T_{\mathrm{m}}$ lies between that of MmTBP37 and MtTBP65 indicating that the interaction between the $\alpha$-helical and $\beta$-sheet part of the TBP is crucial for the thermal stability. The temperature stability is probably encoded in the variable $\alpha$-helices that interact with the highly conserved and DNA binding $\beta$-sheets.
\end{abstract}

Keywords: TATA box binding protein, hybrid, archaea, thermoadaptation, protein stability, infrared spectroscopy

\section{Abbreviations}

TBP TATA box binding protein;

MmTBP37 TBP from Methanosarcina mazei;

MtTBP65 TBP from Methanothermobacter thermautotrophicus;

$T_{\mathrm{m}} \quad$ transition temperature;

OGT optimal growth temperature;

FTIR Fourier transform infrared.

\section{Introduction}

The stability of proteins at the respective growth temperature of the host species is crucial for all processes of life. Its origins are therefore an important field of protein research. Different factors have

\footnotetext{
${ }^{*}$ Corresponding author: Karin Hauser, Institute of Physics, RWTH Aachen University, Sommerfeldstr. 14, 52074 Aachen, Germany. Tel.: +49 241 8027164; Fax: +49 241 8022331; E-mail: hauser@ physik.rwth-aachen.de.
} 


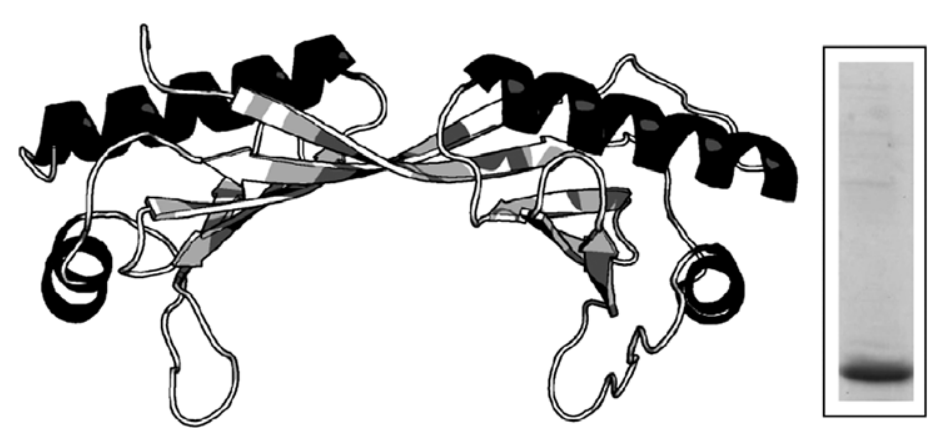

Fig. 1. Model of the tertiary structure of Hybrid-TBP. The $\alpha$-helices are originating from MmTBP37 (black) and the $\beta$-sheets from MtTBP65 (white). The model structure was generated with Swiss-model [1]. The gel shows the purity of the Hybrid-TBP analyzed via SDS-PAGE.

been found so far that influence especially the thermal stability, like the number of hydrogen bonds or salt bridges, more dense packing of secondary structure or dimerization [4]. A promising approach to understand thermoadaptation is the comparative analysis of homologous proteins with nearly identical structure but different thermal stabilities. The TATA Box Binding protein (TBP) is ideally suited for this kind of study. It is a $20 \mathrm{kD}$ protein consisting of two repeating sequences of about 100 amino acids with a similarity of $\sim 40 \%$ (eukaryotic TBPs contain a third unrelated domain of varying length at the $\mathrm{N}$-terminus). It has a saddle-shaped structure consisting of two $\alpha$-helices that lie above four antiparallel $\beta$-sheets and two small $\alpha$-helices located at the sides (Fig. 1). The protein consists of about $25 \%$ $\alpha$-helices and 33\% $\beta$-sheet. The $\beta$-sheets form the DNA binding domain of the protein and are therefore essential for its function. The TBP structure remains the same with and without DNA as revealed by the currently available crystal structures $[3,7,8]$. Most importantly it is universally conserved and essential in both eucaryots and archaea. Archaeal proteins are especially interesting as many archaeal organisms live in extreme environments like high salt, low $\mathrm{pH}$ or temperatures from below $0^{\circ} \mathrm{C}$ as well as more than $100^{\circ} \mathrm{C}$. Among archaea, the TBP has a similarity of $40-55 \%$. Several studies analyzed the thermostability of archaeal TBP indicating that the temperature stability of a TBP is related to the optimal growth temperature of its donor organism $[3,5,6]$. Thus the TBP family consists of proteins with high structural identity but very different temperature stabilities. In this study, a "new" TBP will be introduced, an artificial protein, that is a hybrid between a mesophilic (Methanosarcina mazei, optimal growth temperature $37^{\circ} \mathrm{C}$, MmTBP37) and a thermophilic TBP (Methanothermobacter thermoautotrophicus, optimal growth temperature $65^{\circ} \mathrm{C}$, MtTBP65). We have previously characterized both TBPs using IR-spectroscopy and determined their transition temperatures $\left(T_{\mathrm{m}}\right)$ [6]. MmTBP37 was the first archaeal mesophilic TBP that proved the $T_{\mathrm{m}}$ - OGT dependence of archaeal TBPs [6]. The Hybrid-TBP presented here consists of MtTBP65 whose $\alpha$-helices were exchanged by those of MmTBP37. This construct was analyzed with IR spectroscopy in order to reveal the influence of the different structural parts on the thermal stability. Furthermore, indications about the interaction between the $\beta$-sheet interface and the $\alpha$-helices were obtained in order to gain insight into the mechanisms of protein thermoadaptation.

\section{Materials and methods}

The hybrid-tbp-gene was constructed via fusion-pcr from mm-tbp37 wild-type and $m t$-tbp 65 wildtype. In essence, six overlapping pcr fragments were generated that were fused into one fragment using 
a series of consecutive pcr reactions. First, fragments 1 and 2 and fragments 5 and 6 were fused together, afterwards the fragment 3 was fused to $1 / 2$, and 4 was fused to $5 / 6$. Finally, both resulting fragments $1 / 2 / 3$ and $4 / 5 / 6$ were fused together to obtain the complete hybrid gene. Thereby the regions encoding the two short and the two long $\alpha$-helices of $m t$-tbp 65 were replaced by the corresponding regions of $\mathrm{mm}$ tbp37. The hybrid gene was ligated into a pQE-30 vector (Qiagen) and its sequence was verified. Protein production and purification were carried out as described before [6]. The following oligonucleotides were used (the nucleotides shown in bold encode part of the $\alpha$-helices of MmTBP37; overlapping regions used for the fusion pcrs are underlined):

\begin{tabular}{llll}
\hline primer1_forw: & cgagctcttgacagatgtggatatcaaaatagaaat & primer1_rev: & attcgataacagttaaatcaatggattttcca \\
primer2_forw: & actgttatcgaatcagagcttgagaatgtt & primer2_rev: & acatcggcaactgattggctcctgta \\
primer3_forw: & ccaaatcagttgccgatgtacacacagt & primer3_rev: & tcaggatctatactgttcagctttttcgc \\
primer4_forw: & aacagtatagatcctgacataccagagg & primer4_rev: & attgcaattgcattaaggttaagtggttttc \\
primer5_forw: & ttaatgcaattgcaataggacttgaaaataca & primer5_rev: & atcttcaggactcttggctctgtaca \\
primer6_forw: & primer6_rev: & cccaagcttttaaaagacccatactgtcgagctgct \\
\hline
\end{tabular}

FTIR measurements at different temperatures were performed with an Equinox 55 spectrometer (Bruker, Ettlingen, Germany). The temperature was controlled with a thermostated water bath connected to a home-built cell holder. The sample was heated and equilibrated in steps of $2^{\circ} \mathrm{C}$. $\mathrm{CaF}_{2}$-cuvettes with an optical path-length of $10 \mu \mathrm{m}$ were used. The samples were diluted in $10 \mathrm{mM} \mathrm{D}_{2} \mathrm{O}$-phosphate buffer at $\mathrm{pH} 7$ with a protein concentration of $25 \mathrm{mg} / \mathrm{ml}$. The transition temperature was determined by the thermally induced frequency shift of the amide $\mathrm{I}^{\prime}$ band of the $\beta$-sheet to the aggregation band. More details about the measurements and the data evaluation have been described previously [6].

\section{Results and discussion}

The Hybrid-tbp gene was constructed from six pcr fragments, which contained parts of the $m m-t b p 37$ and $m t$-tbp 65 genes, as explained in Section 2. The hybrid gene was cloned and sequencing revealed that one of the small $\alpha$-helices at the side had not been exchanged. As this small $\alpha$-helix represents only $16 \%$ of the $\alpha$-helix amount or $4 \%$ of the total protein, the construct was used for expression. After expression and purification of the protein, its size and purity were analyzed via SDS-Page. The purity of the isolated protein exceeded $90 \%$ (Fig. 1) and it had the expected size of $22 \mathrm{kDa}$ (data not shown).

Temperature-dependent FTIR measurements have been performed with the Hybrid-TBP in accordance to the measurements of MmTBP37 and MtTBP65 we have done previously [6]. The IR spectra of the Hybrid-TBP clearly showed that the exchange of the $\alpha$-helices of MtTBP65 to those of MmTBP37 results in a folded protein TBP structure consisting of $\alpha$-helices and $\beta$-sheets. We analyzed the second derivative of the IR absorption spectra since they reflect structural changes upon heating with high sensitivity (Fig. 2). At low temperatures, the protein was folded. The band at $1632 \mathrm{~cm}^{-1}$ as well as the weaker band at $\sim 1680 \mathrm{~cm}^{-1}$ could clearly be assigned to the $\beta$-sheet structure. The absorption of the $\alpha$-helical structure was less pronounced and tentatively assigned to the band at $\sim 1650 \mathrm{~cm}^{-1}$. In addition to IR spectroscopy, CD spectroscopy was performed to verify that the $\alpha$-helices had formed in the hybrid protein (data not shown). Upon heating, the amide $\mathrm{I}^{\prime}$ bands of the folded structure decreased and a band at $1618 \mathrm{~cm}^{-1}$ occurred in the FTIR spectrum indicating the aggregation of the protein. This band remained upon cooling. The transition temperature $\left(T_{\mathrm{m}}\right)$ was determined accordingly to the measure- 

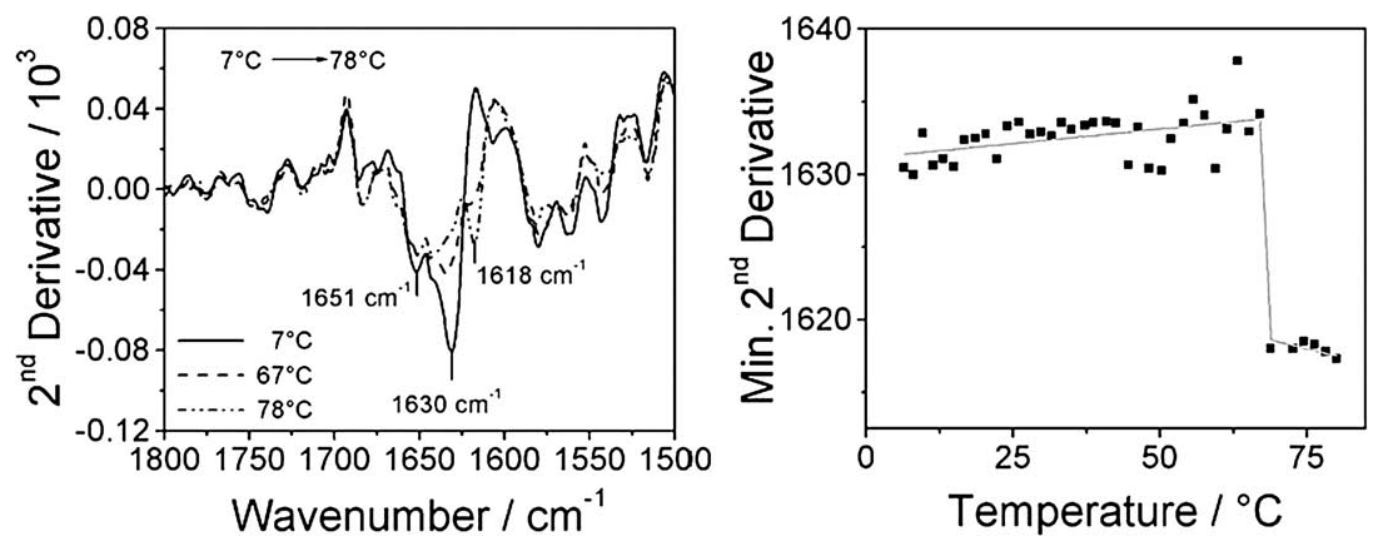

Fig. 2. Determination of the thermal stability of the Hybrid-TBP. Left: Temperature dependent second derivative IR spectra shown for the folded protein $\left(7^{\circ} \mathrm{C}\right)$, the aggregated protein $\left(78^{\circ} \mathrm{C}\right)$ and at the transition temperature $\left(67^{\circ} \mathrm{C}\right)$. Right: The transition curve is obtained by plotting the amide $\mathrm{I}^{\prime}$ frequency shift versus temperature.

ments of MmTBP37 and MtTBP65 [6] by plotting the thermally induced frequency shift of the amide I' $\beta$-sheet component $\left(\sim 1632 \mathrm{~cm}^{-1}\right)$ to the aggregation band $\left(\sim 1620 \mathrm{~cm}^{-1}\right)$. The frequency positions were deduced from the peak minima of the second derivative spectra at each recorded temperature. The $T_{\mathrm{m}}$ of the Hybrid-TBP was determined as $68^{\circ} \mathrm{C}$ at the used protein concentration of $25 \mathrm{mg} / \mathrm{ml}$.

Aggregation may depend on the protein concentration as we could also show for MmTBP37 and MtTBP65, but the aggregation behavior is different for each of the TBPs [6]. At concentrations below $10 \mathrm{mg} / \mathrm{ml}$, the MmTBP37 aggregates $\left(T_{\mathrm{m}}=46^{\circ} \mathrm{C}\right)$, whereas MtTBP65 does not aggregate at all. At $15 \mathrm{mg} / \mathrm{ml}$, the $T_{\mathrm{m}}$ for MmTBP37 increases to $56^{\circ}$ and MtTBP65 aggregates at $69^{\circ} \mathrm{C}$. By further increase of the protein concentration, one obtains transition temperatures of $58^{\circ} \mathrm{C}(\mathrm{MmTBP} 37)$ and $74^{\circ} \mathrm{C}$ (MtTBP65), respectively. As the $T_{\mathrm{m}}$ does not change anymore for concentrations of $20 \mathrm{mg} / \mathrm{ml}$ and above in the case of both TBPs, the study of the Hybrid-TBP was also done at high protein concentrations in order to exclude concentration effects. Thus, at high protein concentrations, the $T_{\mathrm{m}}$ of Hybrid-TBP $\left(68^{\circ} \mathrm{C}\right)$ lies between that of $\mathrm{MmTBP} 37\left(58^{\circ} \mathrm{C}\right)$ and $\mathrm{MtTBP} 65\left(74^{\circ} \mathrm{C}\right)$.

The $T_{\mathrm{m}}$ values are determined by the transition of the $\beta$-sheets to the aggregated protein. Obviously, the interactions of the $\alpha$-helices with the $\beta$-sheets are influencing the transition as the $T_{\mathrm{m}}$ of the Hybrid-TBP is lower than that of MtTBP65. The MmTBP37 $\alpha$-helices seem to destabilize the MtTBP65 $\beta$-sheets as the Hybrid-TBP aggregates before the temperature reaches $74^{\circ} \mathrm{C}$, the $T_{\mathrm{m}}$ of MtTBP65. Thus the exchange of $25 \%$ of the protein leads to destabilization as its $T_{\mathrm{m}}$ decreased from $74^{\circ} \mathrm{C}$ to $68^{\circ} \mathrm{C}$. By comparing the sequences of MmTBP37 and MtTBP65 it becomes obvious that most of the differences in both sequences are located in the $\alpha$-helices and loops whereas the $\beta$-sheet part is highly conserved. The $\beta$-sheet interface is the DNA binding part of the TBP and is essential to maintain its function, thus mutations in this part are probably lethal. This leads to the conclusion that the temperature stability differences of the TBPs from species with different OGTs are probably encoded in the variable $\alpha$-helices that interact with the conserved $\beta$-sheets. Ongoing studies focus on further analysis of the spectra in order to separate the thermostability of the $\beta$-sheet and $\alpha$-helix part and to get deeper insights into the interactions between the structural parts of TBP. It will be interesting to analyze the thermostability of the vice-versa mutant with $\alpha$-helices from MtTBP65 and the $\beta$-sheets from MmTBP37 and to characterize the influence of point mutations. 


\section{References}

[1] K. Arnold, L. Bordoli, J. Kopp and T. Schwede, The SWISS-MODEL workspace: A web-based environment for protein structure homology modelling, Bioinformatics 22 (2006), 195-201.

[2] L. Brocchieri and S. Karlin, Protein length in eukaryotic and prokaryotic proteomes, Nucleic Acids Res. 33 (2005), 33903400.

[3] B.S. DeDecker, R. O’Brien, P.J. Fleming, J.H. Geiger, S.P. Jackson and P.B. Sigler, The crystal structure of a hyperthermophilic archaeal TATA-box binding protein, J. Mol. Biol. 264 (1996), 1072-1084.

[4] P.A. Fields, Review: Protein function at thermal extremes: balancing stability and flexibility, Comp. Biochem. Physiol. A Mol. Integr. Physiol. 129 (2001), 417-431.

[5] H. Koike, Y. Kawashima-Ohya, T. Yamasaki, L. Clowney, Y. Katsuya and M. Suzuki, Origins of protein stability revealed by comparing crystal structures of TATA binding proteins, Structure 12 (2004), 157-168.

[6] A. Kopitz, J. Soppa, C. Krejtschi and K. Hauser, Differential stability of TATA box binding proteins from archaea with different optimal growth temperatures, Spectrochim. Acta A Mol. Biomol. Spectrosc. 73 (2009), 799-804.

[7] O. Littlefield, Y. Korkhin and P.B. Sigler, The structural basis for the oriented assembly of a TBP/TFB/promoter complex, Proc. Natl. Acad. Sci. 96 (1999), 13668-13673.

[8] A.K. Taggart and B.F. Pugh, Dimerization of TFIID when not bound to DNA, Science 272 (1996), 1331-1333. 


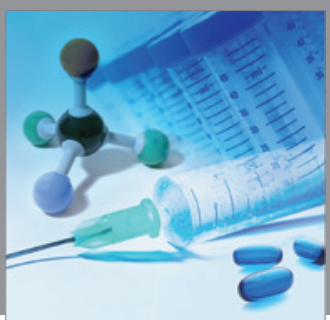

International Journal of

Medicinal Chemistry

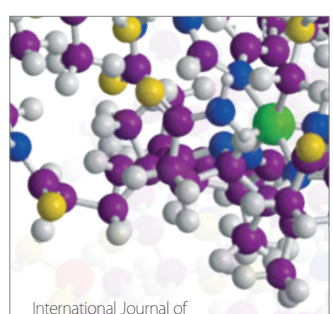

Carbohydrate Chemistry

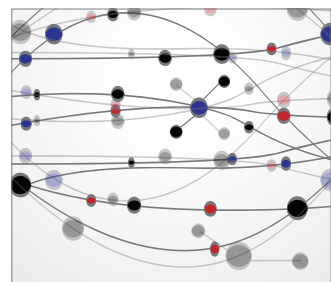

The Scientific World Journal
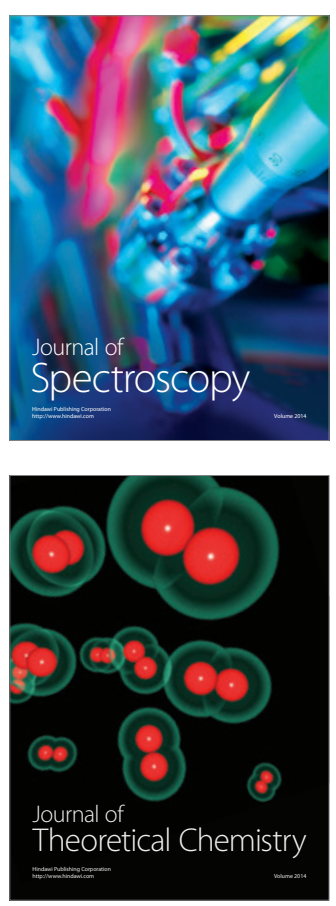
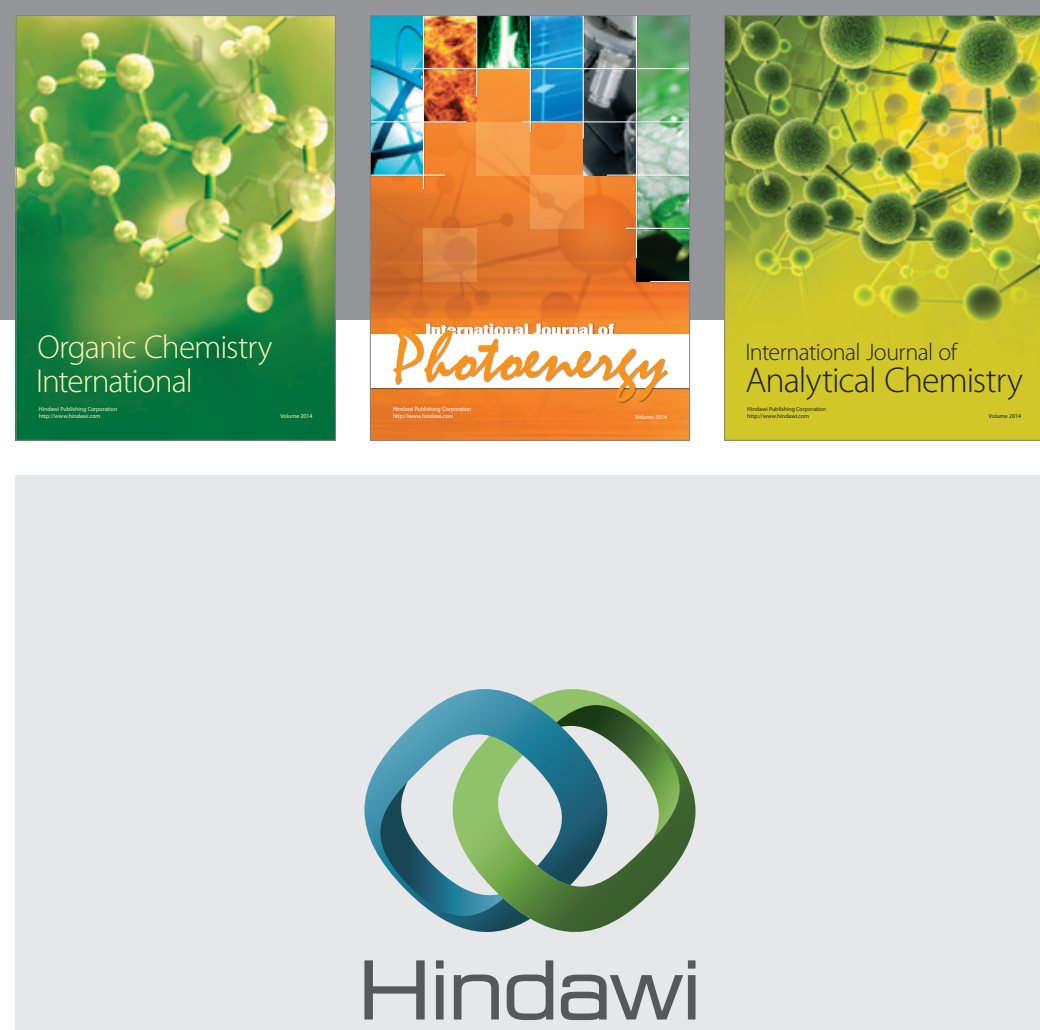

Submit your manuscripts at

http://www.hindawi.com
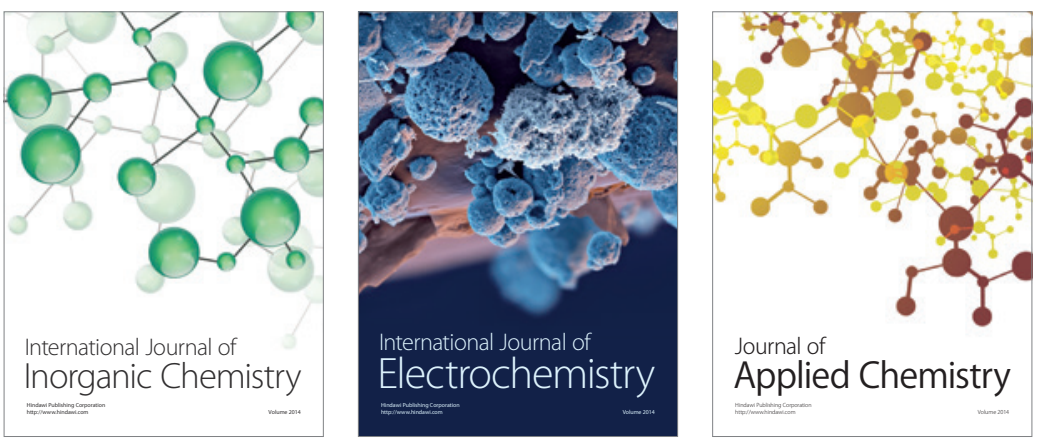

Journal of

Applied Chemistry
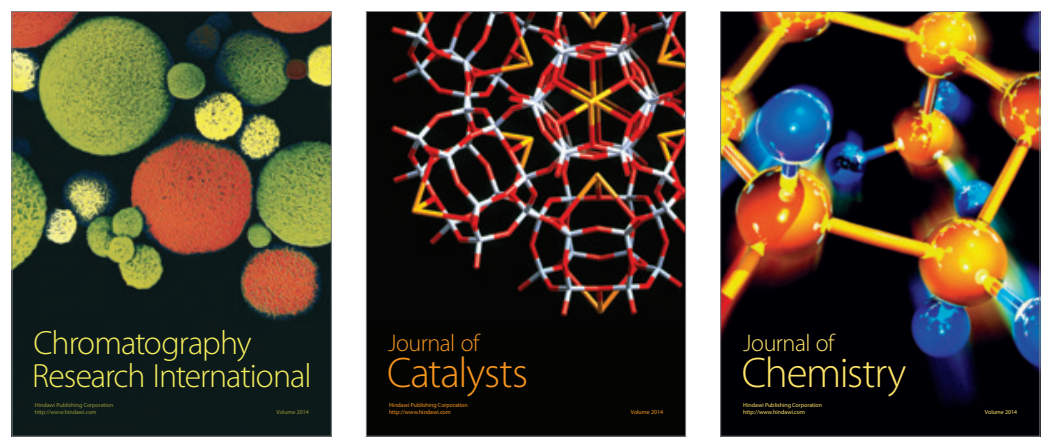
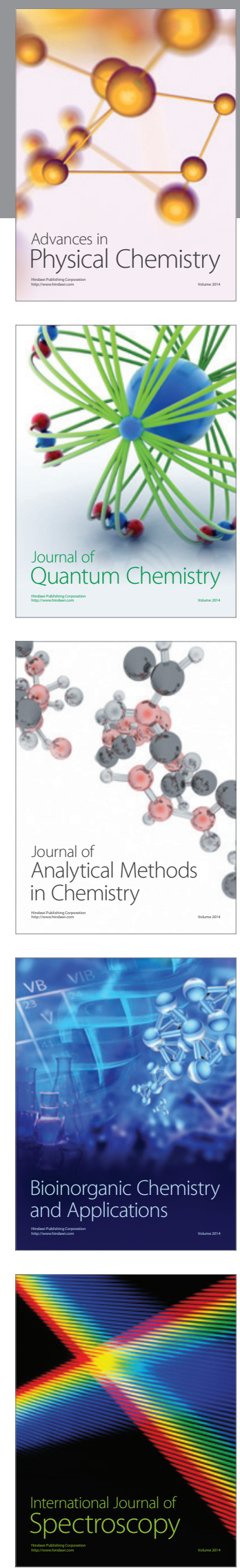